\title{
Identificação do Nível de Conhecimento sobre Aspectos Nutricionais Relacionados ao Câncer em Acadêmicos de Educação Física do lesgf
}

\author{
Determination of the Level of Knowledge about Nutritional Aspects of Cancer among Physical Education Students at lesgf \\ Identificación del Nivel de Conocimiento acerca de los Aspectos Nutricionales Relacionados al Cáncer en \\ Académicos de Educación Física del lesgf
}

Jarvis Duessmann Lembeck'; Heloise Elena de Simas²; Moacir Pereira Junior ${ }^{3}$

Resumo

Introduçáo: $\mathrm{O}$ câncer é uma das principais causas de morte no mundo e é uma das doenças que mais cresce no Brasil. A estimativa é de que 600 mil casos novos sejam diagnosticados para o biênio 2018-2019, em cada ano. Objetivo: O presente estudo tem como objetivo avaliar o nível de conhecimento sobre aspectos nutricionais relacionados ao câncer em acadêmicos de educação física do Instituto de Ensino Superior da Grande Florianópolis. Método: Estudo do tipo descritivo de caráter quantitativo, de corte transversal, com 186 acadêmicos do curso de educação física devidamente matriculados, maiores de 18 anos e que assinaram o Termo de Consentimento Livre e Esclarecido. A caracterização da amostra foi obtida por meio da ficha de identificação, anexada ao instrumento de avaliação. Para avaliar o conhecimento nutricional, os participantes responderam à Escala de Conhecimento Nutricional, composta por 12 questôes, com a pontuação máxima de 14 pontos. Pontuaçóes totais entre 0 e 6 (baixo conhecimento nutricional); entre 7 e 10 (moderado conhecimento nutricional) e acima de 10 (alto conhecimento nutricional). Resultados: $\mathrm{O}$ conhecimento nutricional da amostra revelou um escore total de 8,26 2 ,49 pontos, com mediana de 8 e moda 7 . O resultado apresentado, de modo geral, foi de (57,5\%) moderado conhecimento entre os estudantes, sendo que a correlaçáo entre os pontos do instrumento e o semestre em curso foi de 0,09 ( $\mathrm{p}=0,18)$, e a correlação de dados entre os pontos do instrumento e a idade foi de 0,07 ( $\mathrm{p}=0,27$ ). Conclusão: Em um contexto geral, os acadêmicos de educaçáo física apresentaram moderado conhecimento nutricional sobre o câncer. Em relação aos iniciantes e concluintes, apresentou-se o resultado de moderado conhecimento sobre aspectos nutricionais relacionados ao câncer, sendo que este último apresentou melhor conhecimento. Na comparação entre sexos, houve moderado conhecimento para ambos, porém observou-se que o sexo feminino apresentou um melhor conhecimento nutricional. Palavras-chave: Neoplasias; Conhecimento; Educaçáo Física e Treinamento.

\begin{abstract}
Introduction: Cancer is one of the leading causes of death worldwide and is one of the fastest growing diseases in Brazil, where it is estimated that the annual number of new cases of cancer during the 2018-2019 biennium will be 600,000. Objective: To evaluate the level of knowledge about nutritional aspects of cancer among physical education students at the Greater Florianópolis Institute of Higher Education. Method: This was a descriptive, quantitative, cross-sectional study involving 186 students duly enrolled in a physical education course All of the students were over 18 years of age and gave written informed consent. The sample was characterized by means of an identification form attached to the assessment instrument. The participants completed a nutritional knowledge scale comprising 12 items with a maximum score of 14 points. Nutritional knowledge was categorized as low if the total score was between 0 and 6 , moderate if it was between 7 and 10, and high if it was above ten. Results: The mean total score on the nutritional knowledge scale was $8.26 \pm 2.49$ points, with median of 8 and a mode of 7 . Of the students evaluated, $57.5 \%$ had a moderate level of nutritional knowledge. The score on the nutritional knowledge scale showed a correlation with the academic year of $0.09(\mathrm{p}=0.18)$ and a correlation with age of $0.07(p=0.27)$. Conclusion: In general, our results show that the majority of physical education students have a moderate level of knowledge about nutritional aspects in cancer. The seniors presented better knowledge than did the freshmen. Although the majority of males and females showed a moderate level of knowledge, the proportion of individuals with a high level of knowledge was higher among the females.

Key words: Neoplasms; Knowledge; Physical Education and Training.
\end{abstract}

\section{Resumen}

Introducción: El cáncer es una de las principales causas de muerte en el mundo siendo responsable y es una de las enfermedades que más crece en Brasil con proyección de 600 mil nuevos casos de cáncer serán diagnosticados para el bienio 2018-2019 cada año. Objetivo: El presente estudio tuvo como objetivo evaluar el nivel de conocimiento acerca de los aspectos nutricionales relacionados al cáncer en académicos de Educación Física del Instituto de Enseñanza Superior de la Grande Florianópolis. Método: Estudio del tipo descriptivo de carácter cuantitativo de corte transversal en 186 académicos del curso de Educación Física debidamente matriculados, mayores de 18 años y que firmar el Término de Consentimiento Libre y Esclarecido. La caracterización de la muestra se obtuvo mediante la ficha de identificación adjunta al instrumento de evaluación. Para evaluar el conocimiento nutricional, los participantes respondieron a la Escala de Conocimiento Nutricional, compuesto por 12 preguntas siendo la puntuación máxima de 14 puntos. Puntuaciones totales entre cero y seis (bajo conocimiento nutricional); entre siete y diez (moderado conocimiento nutricional) y por encima de diez (alto conocimiento nutricional). Resultados: El conocimiento nutricional de la muestra reveló una puntuación total de 8,26 2,49 puntos, con mediana de 8 y de la moda 7 . El resultado presentado en general, fue de $(57,5 \%)$ moderado conocimiento entre los estudiantes, y la correlación entre los puntos del instrumento y el semestre en curso fue de $0,09(\mathrm{p}=0,18)$ y la correlación de datos entre puntos del instrumento y edad fue de $0,07(\mathrm{p}=0,27)$. Conclusión: La evaluación del nivel de conocimiento nutricional en académicos de Educación Física, los resultados presentaron moderado conocimiento nutricional. En relación a los iniciantes y concluyentes se presentó el resultado de moderado conocimiento, siendo que este último presentó mejor conocimiento. La comparación entre sexos se presentó moderado conocimiento para ambos, pero se observó que el sexo femenino presentó un mejor conocimiento nutricional.

Palabras clave: Neoplasias; Conocimiento; Educación y Entrenamiento Físico.

\footnotetext{
${ }^{1}$ Bacharel em Educação Física pelo Instituto de Ensino Superior da Grande Florianópolis (lesgf). São José (SC), Brasil. E-mail: jarvislembeck@gmail.com.

${ }^{2}$ Bacharel em Educação Física pelo lesgf. São José (SC), Brasil. E-mail: helo.1994@hotmail.com.

${ }^{3}$ Mestre em Ciências do Movimento Humano. Universidade do Estado de Santa Catarina (Udesc). São José (SC), Brasil. E-mail: moacirpj@gmail.com.

Endereço para correspondência: Moacir Pereira Junior. Rua Doralice Ramos de Pinho, 262 - apartamento 302 - Jardim Cidade. São José (SC), Brasil. CEP 88111 -310.
} 


\section{INTRODUÇÃO}

O câncer é uma enfermidade que se caracteriza pelo crescimento desordenado de células que podem invadir tecidos e órgáos adjacentes e/ou espalharem-se para outras regióes do corpo ${ }^{1-2}$. Existem diversas condições que predispóem uma pessoa ao maior risco de desenvolvimento de câncer, como o meio ambiente, hábitos ou costumes próprios de um ambiente social e cultural, ou internos, resultante de eventos que geram mutaçóes sucessivas no material genético das células, processo que pode ocorrer ao longo de décadas, em múltiplos estágios ${ }^{2}$.

O câncer é uma das principais causas de morte no mundo, sendo responsável por 8,2 milhôes de mortes em 2012 em todo o planeta e 8,8 milhóes em $2015^{3}$. É uma das doenças que mais cresce no Brasil. A estimativa é de que 600 mil casos novos de câncer sejam diagnosticados para o biênio 2018-2019, em cada ano ${ }^{4}$; e, para 2030, esperam-se 27 milhóes de casos novos de câncer em todo o mundo, dos quais, 17 milhôes de pessoas podem morrer de câncer ${ }^{3}$.

Pessoas que consomem, frequentemente, grandes quantidades de carne vermelha e baixas quantidades de carnes brancas apresentam até $50 \%$ de chance a mais para o desenvolvimento do câncer de cólon e reto, em ambos os gêneros ${ }^{2}$. Carnes processadas, como linguiças, salsichas, bacon, entre outras, também aumentam o risco da doença ${ }^{2}$. Os alimentos industrializados, principalmente os embutidos, possuem substâncias usadas como conservantes que contêm importantes agentes cancerígenos, responsáveis pelos altos índices de câncer de estômago, quando consumidos com frequência e em grande quantidade ${ }^{5-8}$.

A alimentação adequada, variada e sem exageros, pode diminuir o risco de desenvolver câncer e, como forma de prevenção, é mais indicada a ingestão de carnes brancas, tais como peixes e aves ${ }^{9-10}$. O farelo de trigo, rico em vitamina $\mathrm{B} 6$, pode reduzir o risco de câncer de pulmão pela metade. $\mathrm{O}$ azeite de oliva e os suplementos de óleo de peixe protegem contra o câncer de mama9 $\mathrm{O}$ consumo de frutas, legumes e verduras, adequado e regular, pode contribuir na redução de $5 \%$ a $12 \%$ dos casos, além de serem ricos em vitaminas e minerais que atuam fortalecendo o sistema imune, atribuindo proteção principalmente contra o câncer de boca, faringe, laringe, esôfago, estômago, pulmão, pâncreas e próstata ${ }^{10}$.

Diante disso, o conhecimento sobre a relação entre os alimentos e nutrientes consumidos e o desenvolvimento do câncer pode promover mudanças no hábito alimentar e levar a escolhas saudáveis, sendo um importante fator na prevenção da doença ${ }^{11}$. Participando nas estratégias de promoção à saúde e prevenção de doenças no meio acadêmico, o conhecimento nutricional representa o processo cognitivo individual relacionado à informação sobre alimentação e nutrição e, ao ser avaliado, permite mensurar a aquisição de informaçôes, possibilitando a elaboração de intervençôes que visam a melhorar os conhecimentos $^{12}$. Portanto, a formação profissional deve ser competente, embasada em conhecimentos, habilidades e atitudes que são adquiridos no decorrer do curso ${ }^{13}$.

Dessa forma, o presente estudo tem como objetivo avaliar o nível de conhecimento sobre aspectos nutricionais relacionados ao câncer em acadêmicos de educação física do Instituto de Ensino Superior da Grande Florianópolis (lesgf).

\section{MÉTODO}

Este estudo é do tipo descritivo de caráter quantitativo, de corte transversal, cuja coleta dos dados aconteceu de 28 a 31 de agosto de 2017. No período do estudo, o curso de educação física do Iesgf possuía 219 acadêmicos devidamente matriculados. Os participantes incluídos para o presente estudo foram os acadêmicos maiores de 18 anos e os que assinaram o Termo de Consentimento Livre e Esclarecido. O critério de exclusão dispensou os acadêmicos que não estavam presentes no dia previsto para a coleta; sendo assim, a amostra inicial do estudo, que era de 219, finalizou com um total de 186 acadêmicos. A caracterização da amostra foi obtida por meio da ficha de identificação, anexada ao questionário, a qual continha informaçóes como idade, sexo, renda familiar, semestre em curso, entre outras informações. A pesquisa seguiu todos os preceitos éticos e foi aprovada pelo Comitê de Ética de Pesquisa em Seres Humanos da Universidade Paulista (Unip), com o número do parecer 2.119.651.

Para avaliar o conhecimento nutricional, os participantes responderam à Escala de Conhecimento Nutricional, a um questionário utilizado para testar o conhecimento nutricional sobre prevenção de câncer, que foi desenvolvido por Harnack et al. ${ }^{14}$ e, posteriormente, traduzido para o português do Brasil e validado por Scagliusi et al. ${ }^{15}$, composto por 12 questóes, com pontuação máxima de 14 pontos. Pontuaçóes totais entre 0 e 6 indicam baixo conhecimento nutricional; entre 7 e 10, indicam moderado conhecimento nutricional; e acima de 10 , indicam alto conhecimento nutricional. A escala compreende questionamentos específicos a respeito da relação entre alimentação, câncer e outras doenças, composição de alimentos ricos em fibras, gorduras e recomendaçôes de frutas e hortaliças.

A coleta dos dados foi realizada por um único pesquisador, aluno do próprio Iesgf, que, no momento da coleta, estava cursando a oitava fase do curso de 
educaçáo física, devidamente treinado. Os participantes foram abordados e avaliados em suas respectivas salas de aula, foram informados do objetivo do estudo, receberam instruçôes de como era a forma de preenchimento do questionário e da ficha com dados referentes à idade, ao sexo e à fase que estava cursando, tendo média de aplicação 15,14 $\pm 3,85$ minutos. A Escala de Conhecimento Nutricional foi autorreferida e as dúvidas foram sanadas antes de iniciar o processo da coleta, para que não obtivesse interferência do pesquisador durante o preenchimento, portanto o instrumento foi autoaplicável.

Os dados foram tabulados em uma planilha Microsoft Excel 2010, foi utilizado o programa estatístico Statistical Package for Social Sciences (SPSS) versão 20.0. A estatística descritiva foi apresentada por meio de frequências absolutas e relativas, média, moda, mediana e desvio-padrão. O teste de normalidade Kolmogorov Smirnov revelou dados não normais, sendo utilizados os testes não paramétricos. $\mathrm{O}$ teste de correlação de Spearman foi utilizado para verificar correlaçấo entre os pontos do questionário e o semestre em curso, e o teste $U$ de Mann Whitney, para comparaçáo entre masculino e feminino. Para todas as análises, considerou-se um nível de significância de 5\%.

\section{RESULTADOS}

A amostra foi composta por 186 acadêmicos de educação física, regularmente matriculados e que atenderam aos critérios de inclusão, sendo 64 mulheres

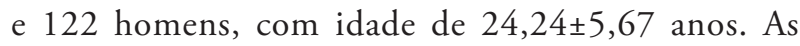
características dos participantes deste estudo estão dispostas na Tabela 1.

Quanto à aplicação do questionário de conhecimento nutricional, o escore total foi de $8,26 \pm 2,49$ pontos, com mediana de 8 e moda 7. De modo geral, observou-se a prevalência da classificação do tipo "moderado

Tabela 1. Características dos acadêmicos do curso de educação física do lesgf, 2017

\begin{tabular}{l|c|c}
\hline \multicolumn{1}{c|}{ Variável } & Categoria & $\boldsymbol{f ( \% )}$ \\
\hline \multirow{3}{*}{ Sexo } & Feminino & $64(34,4)$ \\
\cline { 2 - 3 } & Masculino & $122(65,6)$ \\
\hline \multirow{4}{*}{$\begin{array}{l}\text { Semestre em } \\
\text { curso }\end{array}$} & Primeiro & $26(14,0)$ \\
\cline { 2 - 3 } & Segundo & $49(26,3)$ \\
\cline { 2 - 3 } & Terceiro & $16(8,6)$ \\
\cline { 2 - 3 } & Quarto & $37(19,9)$ \\
\cline { 2 - 3 } & Quinto & $10(5,4)$ \\
\cline { 2 - 3 } & Sexto & $35(18,8)$ \\
\cline { 2 - 3 } & Oitavo & $13(7,0)$ \\
\hline
\end{tabular}

f=número de participantes. conhecimento nutricional" sobre aspectos nutricionais relacionados ao câncer. Os dados estão dispostos na Tabela 2.

Tabela 2. Distribuição da pontuação geral dos acadêmicos do curso de educação física do lesgf, 2017

\begin{tabular}{l|c|c}
\hline \multicolumn{1}{c|}{ Pontuação } & $\begin{array}{c}\text { Classificação do } \\
\text { conhecimento } \\
\text { nutricional }\end{array}$ & $\mathbf{f}(\%)$ \\
\hline > que 10 pontos & Alto & $37(19,9)$ \\
\hline De 7 a 10 pontos & Moderado & $107(57,5)$ \\
\hline De 0 a 6 pontos & Baixo & $42(22,6)$ \\
\hline
\end{tabular}

$\mathrm{f}=$ número de participantes.

A porcentagem de iniciantes e concluintes com baixo, moderado e alto conhecimento nutricional sobre a doença encontra-se no Gráfico 1. De modo geral, observa-se que tanto iniciantes que são acadêmicos do primeiro ao quarto semestre em curso, quanto concluintes que são acadêmicos do quinto ao oitavo semestre em curso, apresentam, em sua maioria, conhecimento nutricional moderado. Entretanto, nota-se que a percentagem é maior no alto conhecimento nutricional dos concluintes, ao contrário do observado nos iniciantes.

O Gráfico 2 ilustra a porcentagem com baixo, moderado e alto conhecimento nutricional sobre a doença entre os sexos. Geralmente, observa-se que tanto o sexo feminino quanto o masculino também apresentam, na maior parte dos casos, conhecimento nutricional moderado. Entretanto, nota-se que a percentagem é maior no alto conhecimento nutricional do feminino, ao contrário do observado no masculino.
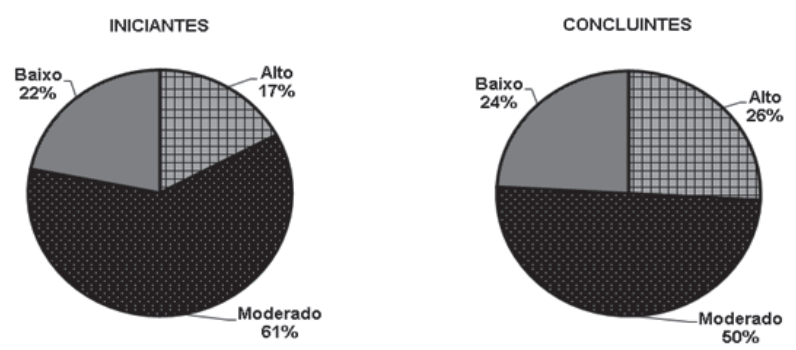

Gráfico 1. Conhecimento nutricional sobre o câncer dos iniciantes e concluintes

Em relação ao conhecimento geral dos participantes, tanto em iniciantes e concluintes quanto no feminino e masculino, os dados apresentaram uma prevalência da classificação do tipo "moderado conhecimento nutricional". 

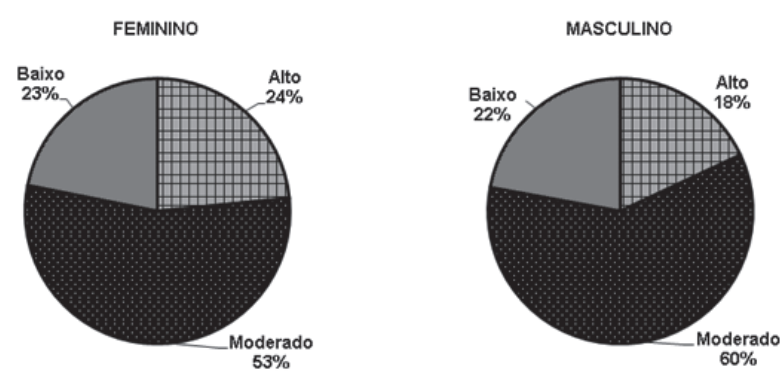

Gráfico 2. Conhecimento nutricional sobre o câncer entre os sexos feminino e masculino

Utilizou-se o teste de Spearman para verificar tanto a correlação entre os pontos do questionário e o semestre em curso, com resultado de $0,09(\mathrm{p}=0,18)$, quanto a correlação entre os pontos do questionário e a idade, com resposta de $0,07(\mathrm{p}=0,27)$. Ambas apresentaram uma correlação positiva fraca.

\section{DISCUSSÃO}

No Brasil, o câncer é a segunda causa de morte diante das demais doenças; e um dos fatores para essa causa é o padrão dietético brasileiro, que parece ter diminuído o consumo de frutas, feijão e vegetais e, por outro lado, aumentado o de bebidas alcoólicas e alimentos de origem animal, seguindo uma dieta que promove a neoplasia ${ }^{6,9}$.

De acordo com dados de pesquisas epidemiológicas, entre as mortes por câncer atribuídas a fatores ambientais, a dieta alimentar contribui com cerca de $35 \%$ dos casos $^{4,5}$; dessa forma, percebe-se que a alimentação é um fator ambiental perfeitamente modificável e que pode reduzir significantemente o número de casos de câncer, uma vez que um terço dos casos de câncer no mundo poderia ser evitado, por meio de dieta alimentar adequada ${ }^{7}$.

Diante dessa premissa, vêm se estabelecendo atividades educativas que se propóem a transmitir informaçóes voltadas para provocar mudanças de hábito de vida da populaçãoo ${ }^{16-18}$. O conhecimento nutricional pode ser definido como um construto científico criado por educadores nutricionais para representar o processo cognitivo individual relacionado à informação sobre alimentação e nutrição; pois, a partir dele, o indivíduo apropria-se do conhecimento e torna-se autônomo para realizar as mudanças necessárias à promoção da sua saúde, sendo esse fator decisivo para a qualidade da dieta alimentar ${ }^{18}$.

Em relação ao conhecimento nutricional, até o momento, a literatura é escassa no que concerne ao uso do presente instrumento para avaliação de conhecimento nutricional em estudantes do ensino superior, especificamente no curso de educação física. $\mathrm{Na}$ validação do instrumento proposto por Scagliusi et al. ${ }^{15}$, observou-se a ocorrência de moderado conhecimento para pacientes com câncer e alto conhecimento para estudantes de nutrição.

O presente estudo buscou identificar o nível de conhecimento nutricional sobre o câncer e fazer correlaçóes entre variáveis e comparaçôes entre grupos, no qual, o resultado apresentado, de modo geral, foi de $(57,5 \%)$ moderado conhecimento entre os estudantes, corroborando os estudos de Gatti et al. ${ }^{19}$, que avaliaram o nível de conhecimento nutricional de acadêmicos, de ambos os sexos, maiores de 18 anos, dos cursos de fisioterapia, psicologia, gastronomia, e educaçáo física. O principal dado encontrado no estudo é que a maioria dos acadêmicos $(82,3 \%)$ também apresentou moderado conhecimento nutricional. No entanto, algumas estratégias devem ser criadas, como projetos de extensão interdisciplinares que envolvam açóes educacionais no combate ao câncer, inclusão de disciplinas curriculares que possam pôr em pauta a importância da discussão da nutriçáo e o câncer, entre outras, para que o conhecimento nutricional demonstrado no ensino superior seja fator determinante para a promoção de hábitos saudáveis e prevenção do câncer ${ }^{19}$.

Alguns estudos obtiveram achados semelhantes com o presente estudo, como Prates e Silva ${ }^{20}$, que avaliaram o conhecimento nutricional e os hábitos alimentares de adultos com doenças crônicas não transmissíveis, e verificaram que a classificação do conhecimento foi moderado, o mesmo achado por Sedó et al. ${ }^{11}$, que, ao avaliarem o conhecimento nutricional de mulheres com câncer de mama, apontaram que $(50,8 \%)$ das entrevistadas apresentaram moderado conhecimento nutricional. Rubin et al. ${ }^{21}$ verificaram o conhecimento nutricional de mulheres sobreviventes de câncer de mama, apresentando o resultado de moderado conhecimento nutricional, no qual verificou-se que o conhecimento nutricional se associou somente à escolaridade; ou seja, quanto maior o grau de instrução das mulheres, maior o conhecimento nutricional.

Como uma das variáveis, o presente estudo comparou o conhecimento nutricional entre acadêmicos iniciantes e concluintes. Entretanto, observou-se que o percentual do alto conhecimento nutricional é maior em concluintes, ao contrário do observado nos iniciantes. Esses resultados corroboram os achados de Nicastro et al. ${ }^{12}$, que avaliaram atletas profissionais e amadores de atletismo, em que a maioria dos atletas profissionais $(53,8 \%)$ se apresentou com o ensino superior incompleto, enquanto atletas amadores $(90,2 \%)$ tinham, substancialmente, concluído o ensino superior. Os resultados mostraram que atletas profissionais e amadores de atletismo apresentaram, de modo geral, conhecimento nutricional moderado, sendo que este último apresentou pontuação significativamente 
maior do que o grupo de profissional, fato este associado ao maior grau de escolaridade; isto é, quanto maior a escolaridade, maior o nível de conhecimento.

Ao avaliar o conhecimento nutricional dos acadêmicos, corroborando os achados dos estudos de Nicastro et al. ${ }^{12}$, Gatti et al. ${ }^{19} \mathrm{e}$ Rubin et al. ${ }^{21}$, a associação entre escolaridade e a pontuaçáo obtida no instrumento de conhecimento nutricional evidencia a importância do acesso à informação e o maior nível de escolaridade como um meio para obtenção de um melhor conhecimento nutricional, como o alcançado pelos acadêmicos concluintes. Esse fator, aliado ao maior grau de instruçấo, pode ser um determinante esclarecedor na correlação positiva entre o tempo de estudos e o conhecimento nutricional.

Ao investigar o nível de conhecimento entre sexos, as mulheres comparadas aos homens apresentaram melhor conhecimento, pois buscam relativamente maiores informaçóes relacionadas à alimentação. De acordo com Zawila et al. ${ }^{22}$, isso pode ser influenciado por alguns fatores, entre eles, os ambientais e culturais.

Em relação à comparação entre homens e mulheres, este estudo mostrou que o sexo feminino obteve um melhor conhecimento nutricional sobre a doença do que o masculino, o mesmo achado nos estudos Nicastro et al. ${ }^{12} \mathrm{e}$ Gatti et al. ${ }^{19}$, em que o sexo feminino também apresentou melhor conhecimento. Esse fato pode ser explicado em razão de as mulheres, em geral, demonstrarem maior preocupação com questóes estéticas, composição corporal e com a saúde, uma vez que elas procuram mais os serviços de saúde e fazem mais exames rotineiros ${ }^{22-23}$.

Embora existam evidências que sugerem que o conhecimento nutricional possa determinar as escolhas alimentares, são necessárias medidas educacionais para estimular a adoção de hábitos alimentares saudáveis e, consequentemente, prevenir o câncer ${ }^{10-11}$. Portanto, propóe-se, para futuros estudos, ampliar a investigaçâo para direcionar açôes efetivas de orientação, de conhecimento sobre o câncer no ensino superior.

Como principais limitaçôes, este estudo se baseou em um desenho transversal e, portanto, não existe uma associação clara entre as variáveis dependentes e independentes. Além de ter sido pautado em um questionário autoaplicável, no qual os participantes poderiam descrever suas ideias percebidas. Por fim, a diferença amostral entre iniciantes e concluintes e entre homens e mulheres, sem a possibilidade de elementos para um tratamento estatístico mais específico.

\section{CONCLUSÃO}

Conclui-se que, em um contexto geral, os acadêmicos de educação física apresentaram moderado conhecimento nutricional sobre o câncer. Em relação aos iniciantes e concluintes, apresentou-se o resultado de moderado conhecimento sobre aspectos nutricionais relacionados ao câncer, sendo que este último obteve melhor conhecimento. Na comparação entre sexos, houve moderado conhecimento para ambos, porém observou-se que o sexo feminino apresentou um melhor conhecimento nutricional.

\section{CONTRIBUIÇÕES}

Jarvis Duessmann Lembeck e Heloise Elena de Simas contribuíram na obtenção e/ou análise e interpretação dos dados. Moacir Pereira Junior contribuiu na concepção e planejamento do projeto de pesquisa.

\section{DECLARAÇÃO DE CONFLITO DE INTERESSES}

Nada a Declarar.

\section{REFERÊNCIAS}

1. Brasil Ministério da Saúde. Glossário temático controle de câncer: projeto de terminologia da saúde. Brasília; 2013. [acesso em 2017 mar 25]. Disponível em: http:// bvsms.saude.gov/bvs/publicacoes/glossariotematico_ controle_cancer.pdf

2. Instituto Nacional de Câncer José Alencar Gomes da Silva. Câncer. Rio de Janeiro; 2017 [acesso em 2017 mar 25]. Disponível em: http://www2.inca.gov.br/wps/ $\mathrm{wcm} /$ connect/cancer/site/oquee

3. World Health Organization. GLOBOCAN 2012: Estimade Cancer incidence, mortality and prevalence worldwide 2012: Lyon; WHO; 2012.

4. Instituto Nacional de Câncer José Alencar Gomes da Silva. Coordenação de Prevençâao e Vigilância. Estimativa 2016: incidência de câncer no Brasil. Rio de Janeiro: INCA; 2015 [acesso 2017 mar 25]. Disponível em: www. inca.gov.br/estimativa/2016/estimativa-2016-v11.pdf

5. Munhoz MP, Oliveira J, Gonçalves RD, Zambon TB, Oliveira LCN. Efeito do exercício físico e da nutrição na prevenção do câncer. Rev. Odont. Araçatuba. 2016; 37(2):09-16.

6. Instituto Nacional de Câncer José Alencar Gomes da Silva. ABC do câncer: abordagens básicas para o controle do câncer / Instituto Nacional de Câncer. Rio de Janeiro; 2011 [acesso 2017 mar 25]. Disponível em: http:// bvsms.saude.gov.br/bvs/publicacoes/abc_do_cancer.pdf

7. Spínola AV, Manzzo IS Rocha CM. As Relaçóes entre Exercício Físico e Atividade Física e o Câncer. ConScientiae. Saúde. 2007;6(1):39-48.

8. Baú FC, Huth A. Fatores de Risco que Contribuem para o Desenvolvimento do Câncer Gástrico e de Esôfago. Ver. Context. Saúde. 2011;11(21):16-24. 
9. Prado BBF. Influência dos hábitos de vida no desenvolvimento do câncer. Ciênc. Cult. 2014;66(1):21-24.

10. Instituto Nacional de Câncer. Açôes de Enfermagem para o Controle do Câncer uma Proposta de Integração Ensino-Serviço. Rio de Janeiro; 2008 [acesso 2017 mar 31]. Disponível em: bvsms.saude.gov.br/bvs/ publicacoes/acoes_enfermagem_controle_cancer.pdf.

11. Sedó KS, Lima CA, Carneiro PCPD, Albuquerque LS, Araújo CO, Castro AS et al. Conhecimento nutricional de mulheres com câncer de mama e sua relação com o estado nutricional. Ver. Bras. Prom. Saúde. 2013;26(1):71-78.

12. Nicastro H, Dattilo M, Santos TR, Padilha HVG, Zimberg IZ, Crispin CA, et al. Aplicação da escala de conhecimento nutricional em atletas profissionais e amadores de atletismo. Ver. Bras. Med. Esporte. 2008;14(3): 205-8.

13. Antunes A.C. Mercado de trabalho e educação física: aspectos da preparaçáo profissional. Rev. Educ. 2007;10(10):141-149.

14. Harnack L, Block G, Subar A, Lane S, Brand R. Association of cancer prevention-related nutrition knowledge, beliefs and attitudes to cancer prevention dietary behavior. J Am. Diet Assoc. 1997;97(9):957-65.

15. Scagliusi FB, Polacow VO, Cordás TA, Coelho D, AlvarengaM, Philippi ST, et al. Tradução, adaptação e avaliação psicométrica da escala de conhecimento nutricional do National Health Interview Survey Cancer Epidemiology. Ver. Nutr. 2006;19(4):425-36.

16. Lima FEL, Latorre MRDO, Costa MJC, Fisberg RM. Diet and cancer in northeast Brazil: evaluation of eating habits and food group consumption in relation to breast cancer. Cad. Saúde Pública. 2008;24(4):820-8.

17. Barbosa LB, Vasconcelos SML, Correia LOS, Ferreira RC. Estudos de avaliação do conhecimento nutricional de adultos: uma revisão sistemática. Ciênc. Saúde Coletiva. 2016;21(2):449-462.

18. Gonçalves T, Carneiro PCPD, Cals PMT, Leão LS, Lima CA, Verde SMML. Qualidade da dieta de mulheres com câncer de mama e sua relação com o conhecimento nutricional e o estado nutricional. Ver. Bras. Mastologia. 2012;22(1):13-20.

19. Gatti RR, Pegoraro J, Barankevicz GB. Conhecimento e Estado Nutricional Estão Associados Cient. Ciênc. Biol. Saúde. 2013;15(esp.):397-400.

20. Prates RE, Silva AC. Avaliação do conhecimento nutricional e de hábitos alimentares de pacientes com doenças crônicas não transmissíveis em hospital particular no sul do Brasil. Ver. Assoc. Bras. Nutr. 2013;1(1):2-21.

21. Rubin BA, Stein AT, Zelmanowicz AM, Rosa DD. Perfil Antropométrico e Conhecimento Nutricional de Mulheres Sobreviventes de Câncer de Mama do Sul do Brasil. Ver. Bras. Cancerologia. 2010; 56(3):303-309.
22. Zawila LG, Steib CM, Hoogenboom B. The female collegiate cross-country runner: nutritional knowledge and attitudes. J Athl. Train. 2003;38(1):64-74.

23. Silva SSBE, Oliveira SFSB, Pierin AMG. O controle da hipertensão arterial em mulheres e homens: uma análise comparativa. Ver. Esc. Enferm. USP. 2016;50(1):50-8.

Recebido em 22/4/2018

Aprovado em 3/7/2018 